\title{
Timber construction of vernacular buildings in Hong Kong
}

\author{
P. P. Ho \\ Department of Architecture, \\ The Chinese University of Hong Kong, Hong Kong
}

\begin{abstract}
Located along the southern coast of China, Hong Kong possesses a large number of buildings erected between the $17^{\text {th }}$ and early $20^{\text {th }}$ centuries in the style of Pearl River Delta vernacular architecture. These buildings come in different scales and of different building types. In a village, it is common to see large ancestral halls with a complicated timber structural system at one end of the scale as well as common houses with simple construction at the other end of the scale. There are also temples erected in locations away from the villages serving the fishing and merchant communities. Monumental architecture in traditional China followed a well defined modular system in determining sizing of structural members, spatial composition and constructional method. Was there a similar modular system adopted in vernacular buildings in Hong Kong? This paper seeks a structural understanding of the construction of vernacular architecture in Hong Kong by comparing between the building types, sites, and scales. It will suggest a system for analyzing the timber structural system and the construction of vernacular architecture in Hong Kong.
\end{abstract}

Keywords: Hong Kong vernacular architecture, ancestral hall, structural system, timber construction, modular structural system.

\section{Introduction}

Hundreds of villages are scattered across the undulating terrain of what is now known as the New Territories, Hong Kong. When these villages were established by migrants from north China, between the $10^{\text {th }}$ and $20^{\text {th }}$ centuries, this coastal region was very remote from cosmopolitan China. Migrants came to settle here to escape from the turmoil that embroiled Central China around Nanjing and Beijing, and founded villages that were by and large self sufficient. Villages in this part of China are very compact settlements, usually occupied by a clan of a 
single surname. In a well established village, there might be up to 500-600 inhabitants while more recent and smaller villages would have around 100 or so inhabitants. Within the clan that occupied the village, there would be inhabitants from different generations. Living in a hierarchical community, villagers observed a strict code of conduct, worshipped the ancestral spirit collectively, and enjoyed a strong sense of clan solidarity. This has resulted in a village consisting of a range of buildings erected for different purposes. Houses are the most numerous, they are small structures with a small courtyard and lined in rows within the village. Interspersed within the neat layers of houses are temples, study halls and ancestral halls.

The architectural language of these buildings originates from a common tradition of Pearl River Delta. This language is constructed by the choice of building material, construction method, structural system, spatial form, and decorations. The characteristics of this vernacular tradition are the solid gable and end walls built with dense grey bricks, dark grey tiles on gabled roof supported by a wooden structure, and decoration applied primarily to wooden members of the building. The degree of elaboration depends very much on the status of the building and the funding available, expressed by the scale and size of buildings, use of material, and decorations. Architecture manifestation between different villages in Hong Kong is not perceptible. This paper will concentrate on examining the structural system of Hong Kong vernacular architecture with the objective of understanding the structural system employed.

\section{Structural system}

Buildings within a village can be classified by their size and complexity into three categories: small houses, small communal buildings and large communal buildings. The basic built form of a small house is a rectangular building covered with a gabled roof. The entrance to the building is found in the middle of its long side. A small courtyard is enclosed to the front of the building by a wall with an entrance gate. There might also be side rooms flanking the courtyard. A typical size house would measure $11.1 \mathrm{~m} \times 9.3 \mathrm{~m}$. A small communal building, which could be a temple, a study hall, or an ancestral hall, is composed of two buildings with a courtyard in between. The front building serves as the entrance and the rear building the main hall. The size of the building complex is determined by its function and funding, one bay or three bays in width, a bay being the space between two columns. Finally, a large building is composed of three buildings separated by two courtyards. The first building is used as an entrance, the second the main hall, and the last the ritual hall. These three buildings, all three bays in width, are lined up in a symmetrical manner along a central axis. (Fig. 1)

The roof of Hong Kong vernacular buildings is constructed with grey tiles placed on wooden battens. The roof is supported on wooden structures. These could be simply round purlins set into the gable walls supporting the battens. This form is commonly seen in humble houses or simple communal buildings. For large communal buildings, the roof structure would consist of closely packed purlins supported on stacked beams with brackets in between. 


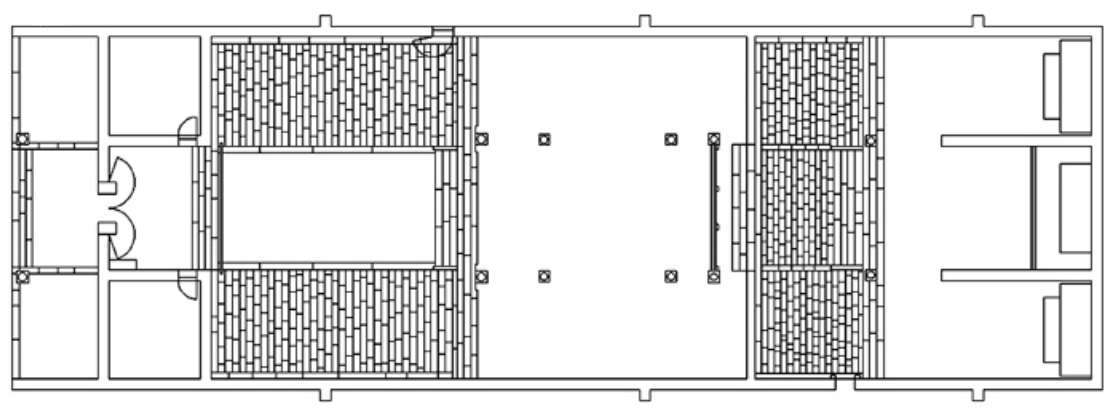

Figure 1: $\quad$ Plan of Tang Chung Ling Ancestral hall, Lung Yeuk Tau, Hong Kong.

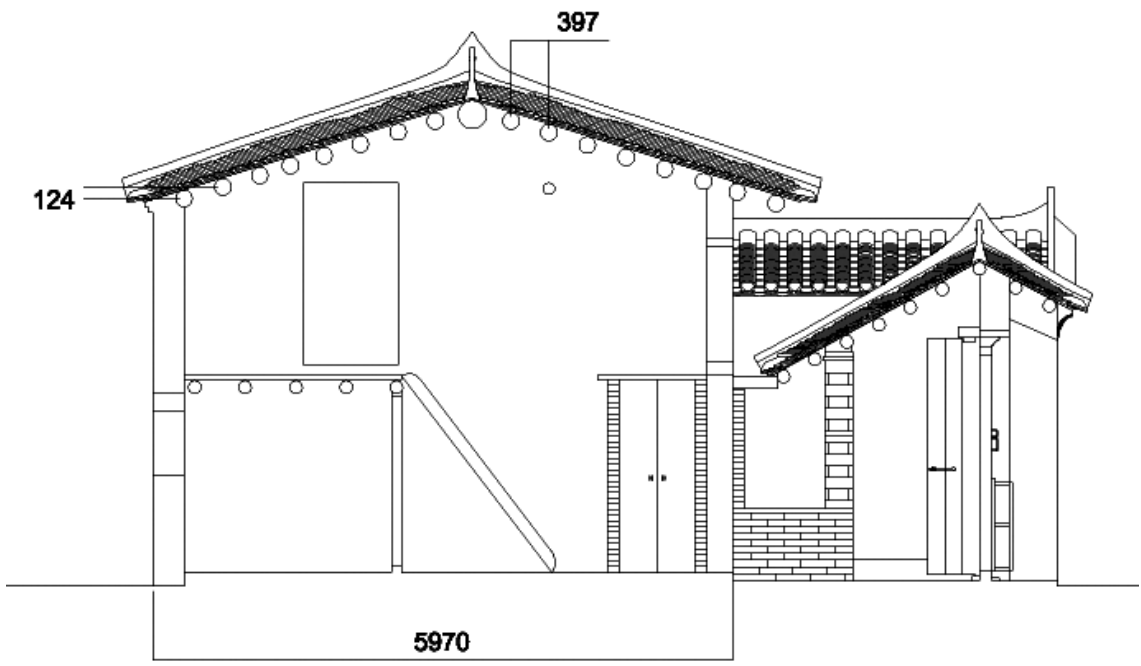

Figure 2: $\quad$ Cheung Village House, Sha Lou Tung, Hong Kong.

\subsection{Small structures}

The simple structure of rafters set inside the wall is probably the most direct form of structure with little room for ornamentation. The size of timber found in the vicinity of Hong Kong is rather small in terms of diameter. Thus it is common to see closely packed purlins used in houses and small structure which has a limited span. One example from a village in Sha Lou Tung is a building measuring 11.13 metres in width by 9.3 metres in depth. (Fig. 2) The main building has a depth of 5.97 metres. The roof structure consists simply of closely packed purlins spanning the width of the main room of around 4.2 metres, resting on internal and gable walls. The diameters of the purlins measure on 
average $170 \mathrm{~mm}$ and spaced at intervals of around $400 \mathrm{~mm}$. The close spacing and diameter of the purlins suggest that the structure is over provided, perhaps for safety reason to prevent buglers climbing through the roof.

\subsection{Medium-size structure}

These are usually structures used in a study hall, a temple or a small ancestral hall. A typical example is the Chik Kwai Study Hall in Pat Heung, built around 1920s. The architectural style is traditional Pearl River Delta vernacular with elaborate decorative elements. The main hall measures 13.4 metres in width and 8.4 metres in depth. The main structure spans a length of 5.2 metres. The roof structure utilizes a composite system. The building is divided into three bays; the roof structure consisting of stacked beams is supported by a granite column on one side and a grey brick wall on the other side. The purlins of the roof structure are spaced at around $550 \mathrm{~mm}$ and are supported on short struts or decorative blocks. The lowest beam has a diameter of $250 \mathrm{~mm}$ and a span of 4.34 metres. The system is referred to as a composite system because of the brick wall used for support on one side. (Right side of Fig. 3)

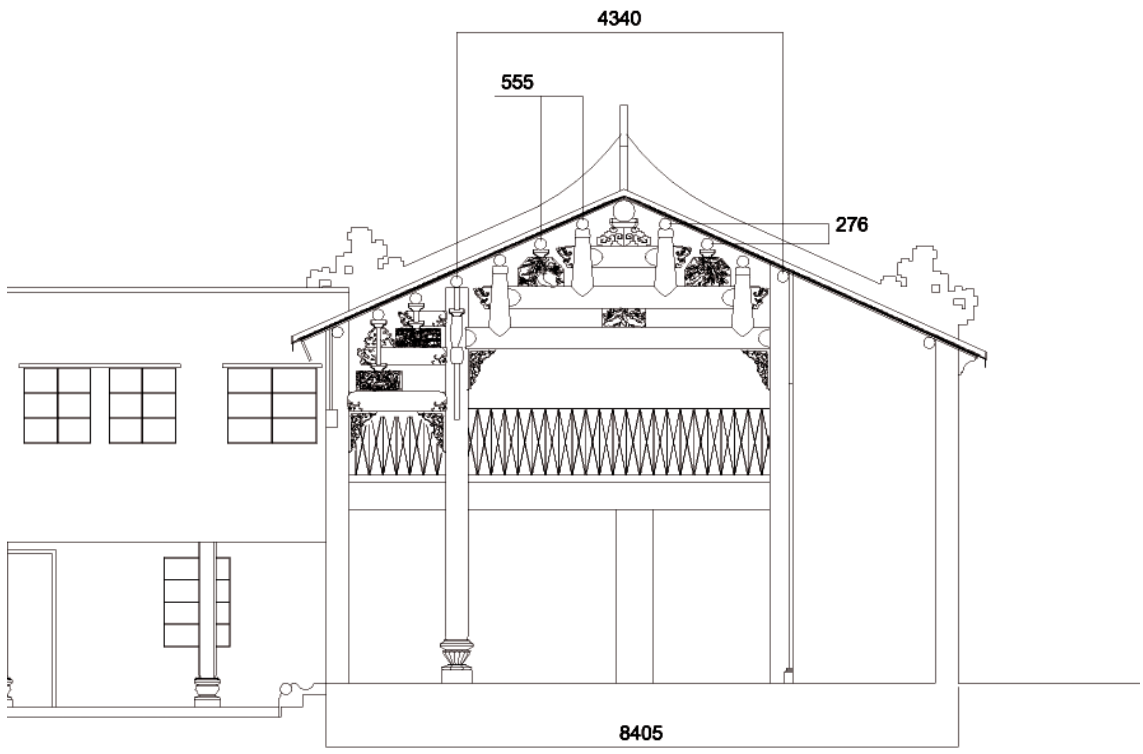

Figure 3: $\quad$ Main hall of Chik Kwai Study Hall, Pat Heung, Hong Kong.

\subsection{Large-size structure}

These structures are used in buildings of a large scale, such as the main hall and rear chamber of a three-building ancestral hall. One good example is the main hall of the Chung Ling ancestral hall of the Tang clan located in Lung Yeuk Tau, dated to $1525 \mathrm{CE}$. The hall measures 11.68 by 15.29 metres, divided into three bays. The main structure employed is a column-and-beam system. The purlins 
are supported on stacked beams that are in turn supported on four columns. The purlins have an average diameter of $250 \mathrm{~mm}$ and spaced at intervals of $890 \mathrm{~mm}$, considerably larger than that of the small or medium size structure. The lowest beam spans 5.34 metres and has a diameter of $270 \mathrm{~mm}$. Decorative blocks are used to separate the cross beams (Fig. 4).

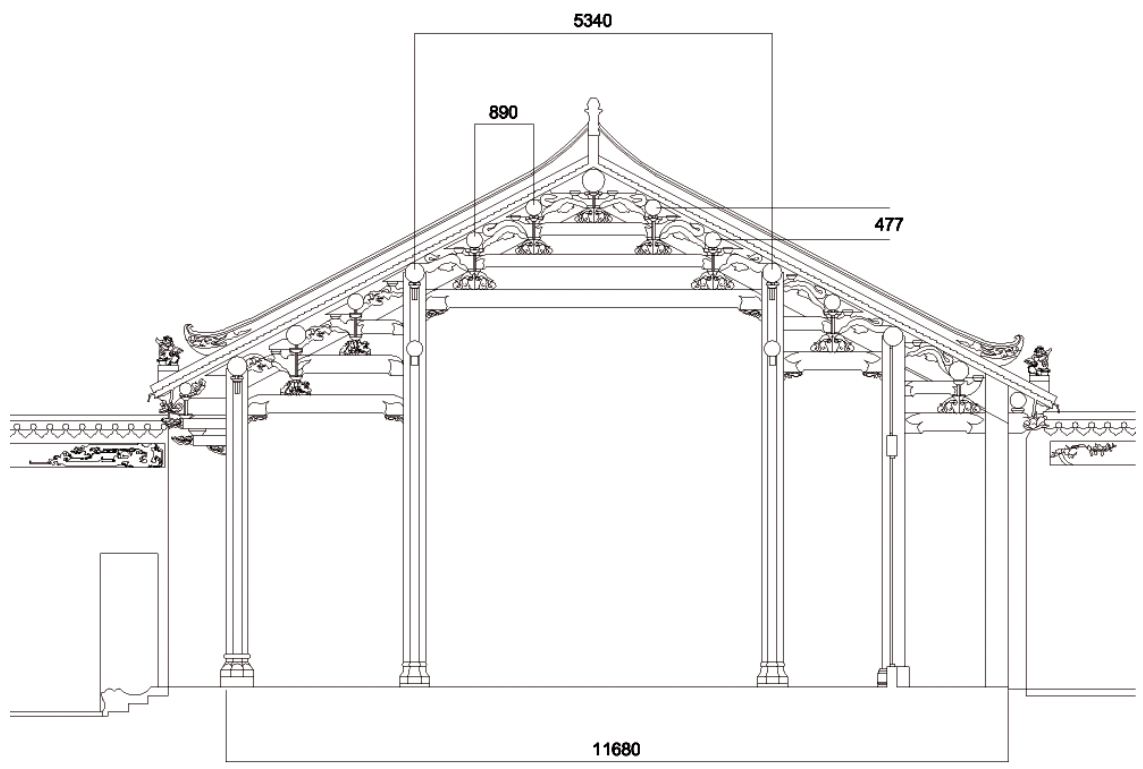

Figure 4: Main hall of Tang Chung Ling Ancestral Hall, Lung Yeuk Tau, Hong Kong.

This large structure can be compared to the main hall of the Bao clan in Tangyue village in southern Anhui province. Many ancestral halls of similar size and construction remain in southern Anhui province. Some of these halls are dated to around $16^{\text {th }}$ century. So for comparison purpose, I have picked the main ancestral hall in Tangyue village, measuring 13.49 by 18.24 metres, a shade larger than common halls in Hong Kong. The Anhui hall has 11 purlins in crosssection, with a spacing of around $1440 \mathrm{~mm}$, while the Hong Kong hall has 13 purlins spaced at $890 \mathrm{~mm}$, thus considerably closer together. The purlins in the Anhui hall are around $150 \mathrm{~mm}$ in diameter for those supporting the roof and hidden from view by the ceiling, spanning 11.6 metres, around $500 \mathrm{~mm}$ for the exposed purlins. Those in Hong Kong hall measure $250 \mathrm{~mm}$ in diameter spanning 6 metres. It is again very clear that the structure used in Hong Kong is more excessive than structural necessary. However, the exposed structure in Anhui hall is clearly built to impressed, the lowest beam has a diameter of $570 \mathrm{~mm}$ spanning 5.7 metres, similar span but twice as large in diameter as that of the Hong Kong hall. The use of large timber in the Anhui hall is probably due to the availability of such timber in the region, while in Pearl River Delta, there was no such supply of large lumber. 


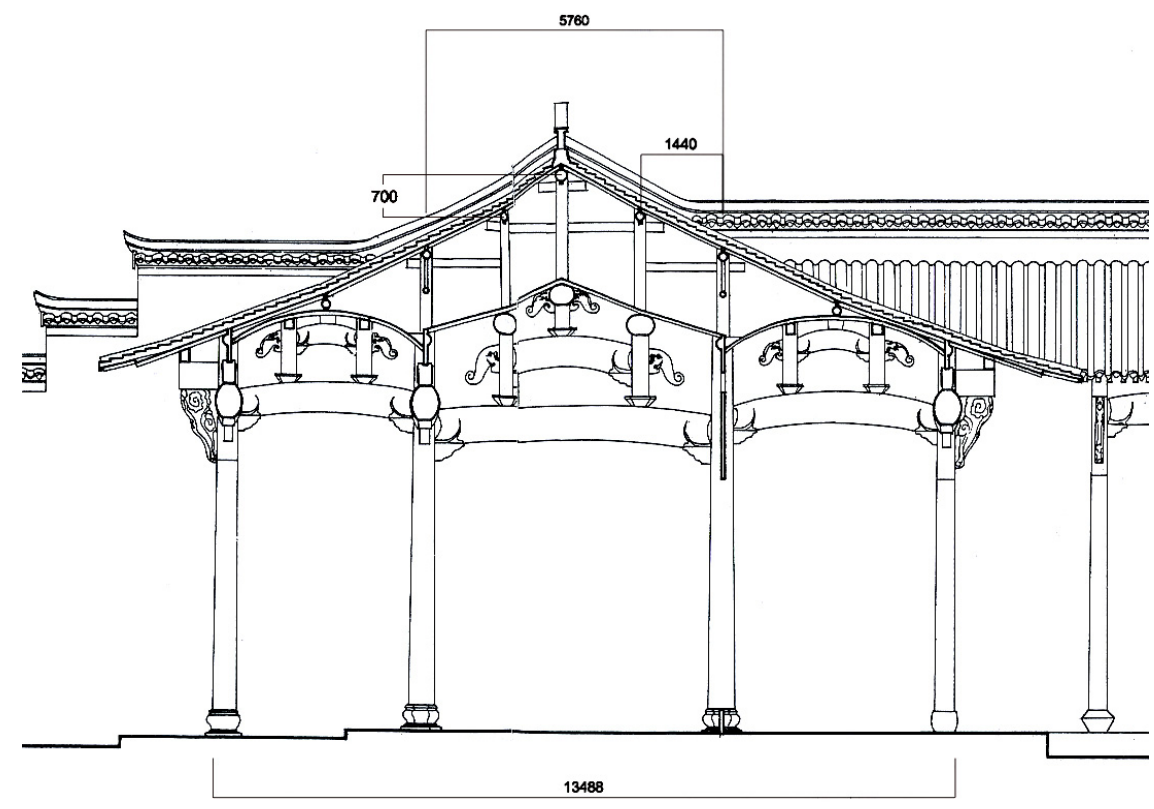

Figure 5: Main Hall of Dun Ben Tang, Tang Yue Village, Huizhou. After The Department of Architecture of Southeast University, Tangyue Series Ancient Architecture in Huizhou (Nanjing: Dongnan daxue chubanshe, 1993).

\section{System of structural design}

How can we make sense of these data for the structure of Hong Kong vernacular architecture? Was there a set of rule governing the choice of structural sizing in Hong Kong? If there is a commonly accepted rule of thumb for structural sizing, what would be the basis for such a rule? For official architecture of the State, there were rule books that are now extant from $1101 \mathrm{CE}$ and the Qing dynasty (1644-1911). Both rule books use a modular system based on the grade of building and the dimension of the bracket block. Simply put, the status and size of buildings will determine its grade for the selection of the dimension of the bracket block. The chosen dimension will then be used for determining the different dimensions of the structure, including the width of the intercolumnal bay, the diameter and length of the columns and other dimensions. This is summarized in Fig. 6 by Ling Sicheng.

However, in vernacular construction, there is not textual evidence of a modular system being used for building in Hong Kong. To be sure, there must be carpentry systems devised for these buildings, the question is whether we can extract the detail of that system based on the study of vernacular buildings? 
From the study narrated above and an analysis of 150 extant buildings, it might be possible to extrapolate the information for suggesting a system of design for vernacular constructions in Hong Kong. For small size buildings, the form of the houses is rather standard in terms of the layout, size of building and the structure member used. For medium-sized buildings, the structural system may be different due to the function of the hall. For a sacrificial hall where there is no need for an enclosed area to house the images of the gods or ancestral tablets, the hall is usually designed which is open on both sides. The purlins are usually supported on stacked beams and in turn supported by a series of columns. For a hall with only one opening, the stacked beams are usually supported on brick wall on the side that is enclosed. The sizes of structural members are again rather standard. This might be due to the availability of materials, such as the girth and length of lumber.

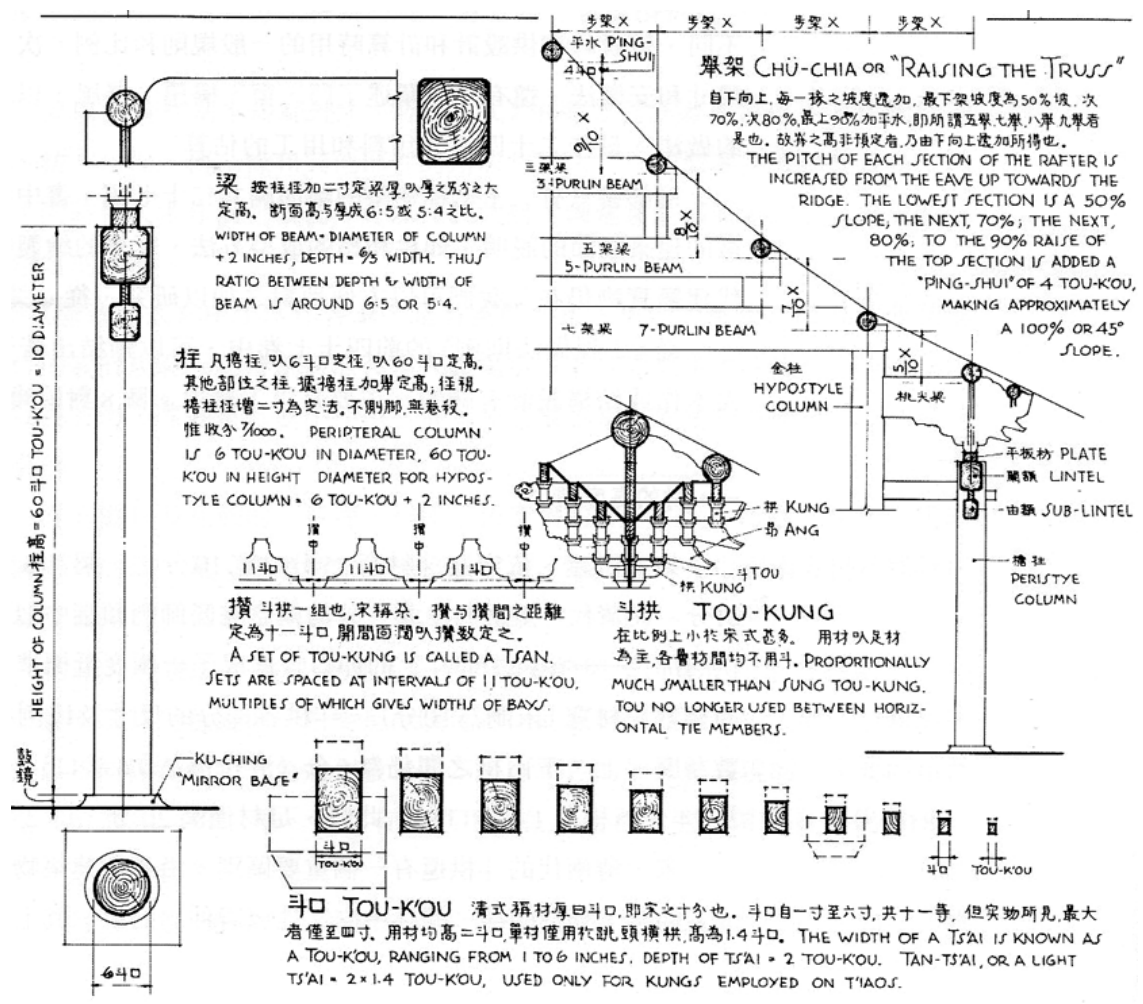

Figure 6: Rules for structural carpentry in Qing dynasty. After Liang Sicheng, A pictorial history of Chinese architecture: a study of the development of its structural system and the evolution of its types (Beijing: Zhongguo jianzhu gongye chubanshe, 1991).

More interesting are the large-size halls. These halls are the largest among wooden buildings in Hong Kong. They enjoyed the highest status and thus were 
constructed to the highest specifications. They are the closest to imperial architectural standard among all vernacular buildings. So they are the closest to the official buildings for comparison. Looking at the selection of 5 major ancestral halls in Hong Kong, they share much similarity in construction and details. In exploring the basis for the structural design of these buildings, I would like to look at a few possibilities (Fig. 6).

The first possibility is that the design of the structural system is based on a modular system. That is, the vernacular system follows the imperial system in which one single dimension will determine the dimensions of the major elements in the timber structure. That single dimension, which is the width of the main block of the bracket set, is determined by the grade of the building in the first place. Thus ancestral hall should qualify as the most important structure in vernacular constructions. However, the bracket set found in the ancestral hall are more for decoration than structural, it is not proper to use the bracket set to determine the modular dimension. If a modular system is indeed used in designing the timber structure, it has to be something else. Several elements might be suitable: the diameter of the eaves column, the diameter of the purlins, or the diameter of cross-beam. However, using these dimensions to form ratios of major dimensions, there is no obvious relational elements. For example, the ratio of column diameter with column height of the main hall yields a range of figures from 11.01 to 15.97 .

Instead of a modular system, the second possibility is that the timber structure is designed with a proportional system between major elements. These elements include the bay width of the building in front elevation, the height of the column along the eaves, height of the eaves purlin or the ridge purlin, or the depth of the inner intercolumnal bay. The diameter of the column and its height can also be a relational set. However, with the data gathered, it is clear that for all five buildings, only a small set of ratios and figures is common to all halls. These include the pitch of the roof, at around 0.25 that is a gradient of one in four. This is related to the other common ratio among the five selected halls, that is the height of the inner eaves purlin and the height of the ridge purlin, which is around 0.81 . The ratio between the height of front eave purlin and the diameter of these columns is not common to all five halls, as described earlier. The ratio of the front eaves column height and the main bay width is again not common. However, the ratio of 1.12 is found in two halls located in two separated villages both related to the Tang clan. It is therefore plausible the main dimensions of the buildings are related to each other. However, based on the current data, it is still not possible to outline the exact proportional relationship.

In summary, this paper sets out to investigate the system of structural design seen in the vernacular architecture of Hong Kong. In particular, the paper attempts to find the basis for determining the sizes and dimensions of the timber structure through a survey of major ancestral halls. It is found that while it is certain that major elements in the structure bear a proportional relationship, it is not sure the exact basis for such relationship. Further research would have to be conducted to determine the method of structural design in Hong Kong's vernacular buildings. 

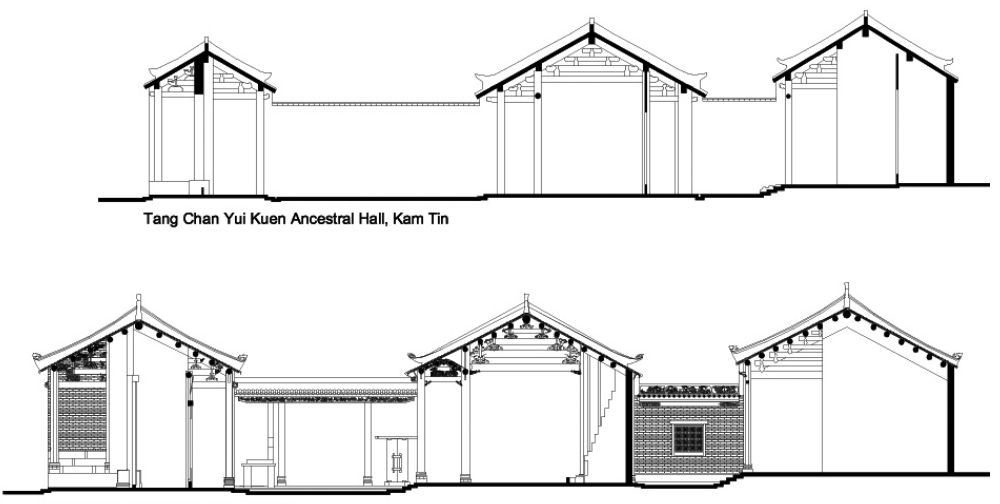

Man Lun Fung Ancestral Hall, San Tin
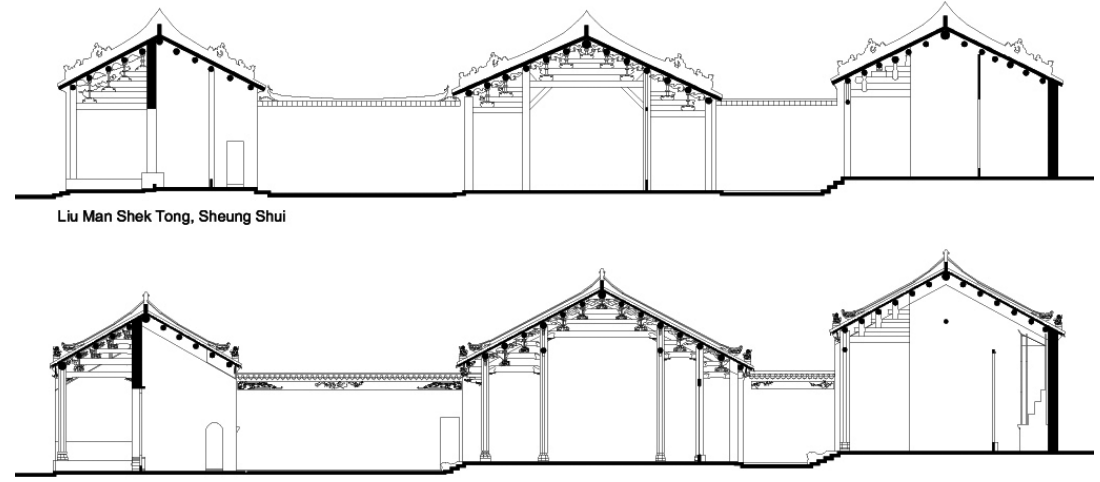

Tang Chung Ling Ancestral Hall, Lung Yeuk Tau

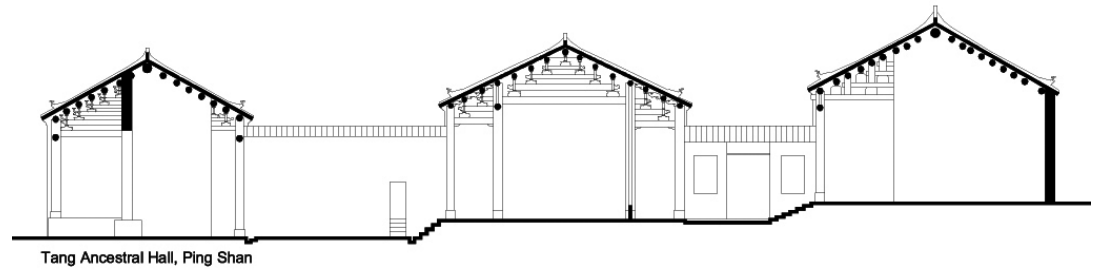

Figure 7: Comparison of the section of 5 major ancestral halls in Hong Kong.

\section{Reference}

[1] Cheng Jianjun: Lingnan gudai dashi diantang jianzhu yanjiu [Study on the Structures of Large Halls of Ancient Buildings in the Lingnan region] (Beijing: Zhongguo jianzhu chubanshe, 2002). 